\title{
Estudo in situ do esmalte dental humano após aplicação de tetrafluoreto de titânio
}

\section{An in situ study of human enamel after titanium tetrafluoride application}

\author{
Andréa Pereira de MORAIS* \\ Ivete Pomarico Ribeiro de SOUZA** \\ Orlando CHEVITARESE***
}

\begin{abstract}
MORAIS, A. P. de; SOUZA, I. P. R. de; CHEVITARESE, O. Estudo in situ do esmalte dental humano após aplicação de tetrafluoreto de titânio. Pesq Odont Bras, v. 14, n. 2, p. 137-143, abr./jun. 2000.

O objetivo deste trabalho, cruzado, duplo-cego, realizado in situ, foi avaliar o esmalte dental humano submetido a um grande desafio cariogênico após aplicação de tetrafluoreto de titânio ( $\left.\mathrm{TiF}_{4}\right)$ a $1 \%$. Para isso, 5 voluntários utilizaram dispositivos intrabucais superiores contendo 80 fragmentos dentais durante 2 etapas ( $\mathrm{TiF}_{4}$ e Controle) de 14 dias cada, gotejando 8 vezes ao dia glicose $25 \%$. Após o uso, o esmalte foi submetido à análise através da MEV (microscopia eletrônica de varredura) e a MDT (microdureza em corte transversal) em 5 posições até a profundidade de $100 \mu \mathrm{m}$ da superficie anatômica do dente. Os resultados não revelaram diferença significativa entre esmalte decíduo e permanente. Os dados da MDT, submetidos à análise de variância multifatorial também não revelaram efeito significativo do $\mathrm{TiF}_{4}$, porém, através da $\mathrm{MEV}$, verificou-se diferença entre $\mathrm{TiF}_{4}$ e Controle com $\mathrm{p}=0,027$ (teste do qui-quadrado). O número de lesões brandas era igual para os dois grupos, porém no Controle haviam 10 fragmentos com esmalte sem cárie e 18 com lesões severas contra 20 e 8 respectivamente do grupo $\mathrm{TiF}_{4}$. Os dados permitem sugerir que o $\mathrm{TiF}_{4} \mathrm{modificou}$ o padrão da lesão formada amenizando-a, sendo necessários novos estudos definindo esse efeito em termos de profundidade.
\end{abstract}

UNITERMOS: Fluoretos tópicos; Titânio; Cárie dentária.

\section{INTRODUÇÃO}

Embora o efeito anticariogênico dos fluoretos tópicos já esteja bem estabelecido, a sua eficácia em relação ao paciente com alta atividade de cárie tem sido questionada. Assim, o interesse em desenvolver agentes anticárie mais efetivos, em especial os que não demandem a cooperação do paciente, continua sendo uma grande preocupação da Odontologia mundial. Por essa razão é que o tetrafluoreto de titânio $\left(\mathrm{TiF}_{4}\right)$, apesar de na comunidade científica não ser um produto novo, vem despertando interesse como agente anticárie.

Os primeiros testes, in vitro, foram realizados em $1972^{18,13}$, e a partir dessa época vários autores como REED; BIBBY ${ }^{17}$ (1976), WEI; SOBOROFF; WEFEL $^{24}$ (1976) e WEFEL; VALERIO; WEI ${ }^{23}$ (1977) reafirmaram as propriedades anticariogênicas do $\mathrm{TiF}_{4}$ em seus estudos, porém a principal preocupação sempre foi elucidar seu mecanismo de ação que levantava dúvidas por se diferenciar da maioria dos fluoretos. Esse mecanismo de ação parece estar intimamente ligado a dois fatos: a formação da apatita fluoretada diminuindo a solubilidade do esmalte, pelo aumento do conteúdo de fluoreto e principalmente, a formação de uma película resistente a ácidos, que pode ser observada através da microscopia eletrônica de varredura $(\mathrm{MEV})$ quando o esmalte é tratado com $\mathrm{TiF}_{4}^{4,7,9,21,22}$. Com finalidade preventiva, essa película pode, inclusive, ser aplicada ao esmalte durante a colagem de bráquetes ortodônticos, representando uma separação distinta entre a superficie do dente e o material de colagem, sem no entanto, diminuir a eficácia do processo $^{1,3,11,12}$.

\section{MATERIAL E MÉTODO Etapa intrabucal}

O delineamento experimental adotado neste es-

\footnotetext{
* Especialista e Mestre em Odontopediatria pela Faculdade de Odontologia da UFRJ. Professora Adjunta do Departamento de Clinica de Pacientes Especiais da UNIGRANRIO.

** Professora Titular do Departamento de Odontopediatria; *** Professor Emérito - Faculdade de Odontologia da UFRJ.
} 
MORAIS, A. P. de; SOUZA, I. P. R. de; CHEVITARESE, O. Estudo in situ do esmalte dental humano após aplicação de tetrafluoreto de titânio. Pesq Odont Bras, v. 14, n. 2, p. 137-143, abr./jun. 2000.

tudo foi do tipo cruzado $2 \times 2$, duplo-cego, constituído de duas etapas de 14 dias cada, e os mesmos voluntários participaram de ambas as etapas.

Em uma das etapas, cinco indivíduos adultos jovens, apresentando bom estado de saúde geral e bucal utilizaram dispositivos intrabucais esterilizados (óxido de etileno) contendo, cada um, quatro fragmentos dentais hígidos oriundos de dentes decíduos e quatro fragmentos de dentes permanentes, todos cobertos por uma rede plástica para acúmulo de placa. Os fragmentos receberam a aplicação de $\mathrm{TiF}_{4}$ a $1 \%(\mathrm{pH} 1,3)$ durante um minuto e durante o uso do dispositivo intrabucal, esses fragmentos foram submetidos a um grande desafio cariogênico (etapa $\mathrm{TiF}_{4}$ ), ou seja, foi gotejada glicose a $25 \%$, oito vezes ao dia durante quatorze dias. $\mathrm{Na}$ outra etapa, após um intervalo de quatro dias, todos os procedimentos foram repetidos, com exceção da aplicação de $\mathrm{TiF}_{4}$ (etapa Controle).

Antes do início da pesquisa, os dentes encontravam-se armazenados em soro fisiológico, e após o corte dos mesmos (Figura 1, cortes A, B e C), de cada quatro fragmentos oriundos de um dente, dois foram escolhidos ao acaso e utilizados na etapa $\mathrm{TiF}_{4}$ e os outros dois na etapa Controle.

Os voluntários receberam orientações para que não fizessem uso de outras formas de aplicação de fluoreto, com exceção da água de abastecimento. Para isso, cada voluntário foi suprido de escova dentária, fio dental e dentifrício sem fluoreto, usa- do para sua escovação diária de rotina. A dieta rotineira de cada voluntário foi mantida nas duas etapas do estudo.

\section{Etapa laboratorial}

Após a etapa intrabucal, os fragmentos foram limpos, retirados dos dispositivos e foram então clivados originando, cada um dos fragmentos, duas amostras; uma foi separada para a observação através da MEV e a outra foi utilizada para a MDT (Figura 1).

Para o estudo através da MEV, as amostras foram posicionadas com sua face interna paralela à base do porta-amostra, ou seja, a região estudada era aquela transversal à superficie externa do esmalte na sua região equatorial (Figuras 1 e 2). Com a finalidade de padronizar o estudo, cada amostra foi dividida em três regiões, e somente o esmalte correspondente à face exposta ao desafio cariogênico foi analisado (Figura 2). Foi utilizado como critério para definir lesão de cárie, o aspecto de total perda da integridade dos prismas de esmalte (intra- e interprismática), consignando ao tecido adamantino uma aparência corroída e porosa característica de um estágio avançado de lesão cario$\mathrm{sa}^{8}$.

Após a primeira observação, as amostras que não possuíam lesões cariosas foram separadas e receberam escore 0 , e as demais foram novamente observadas ao microscópio, nesse momento cada

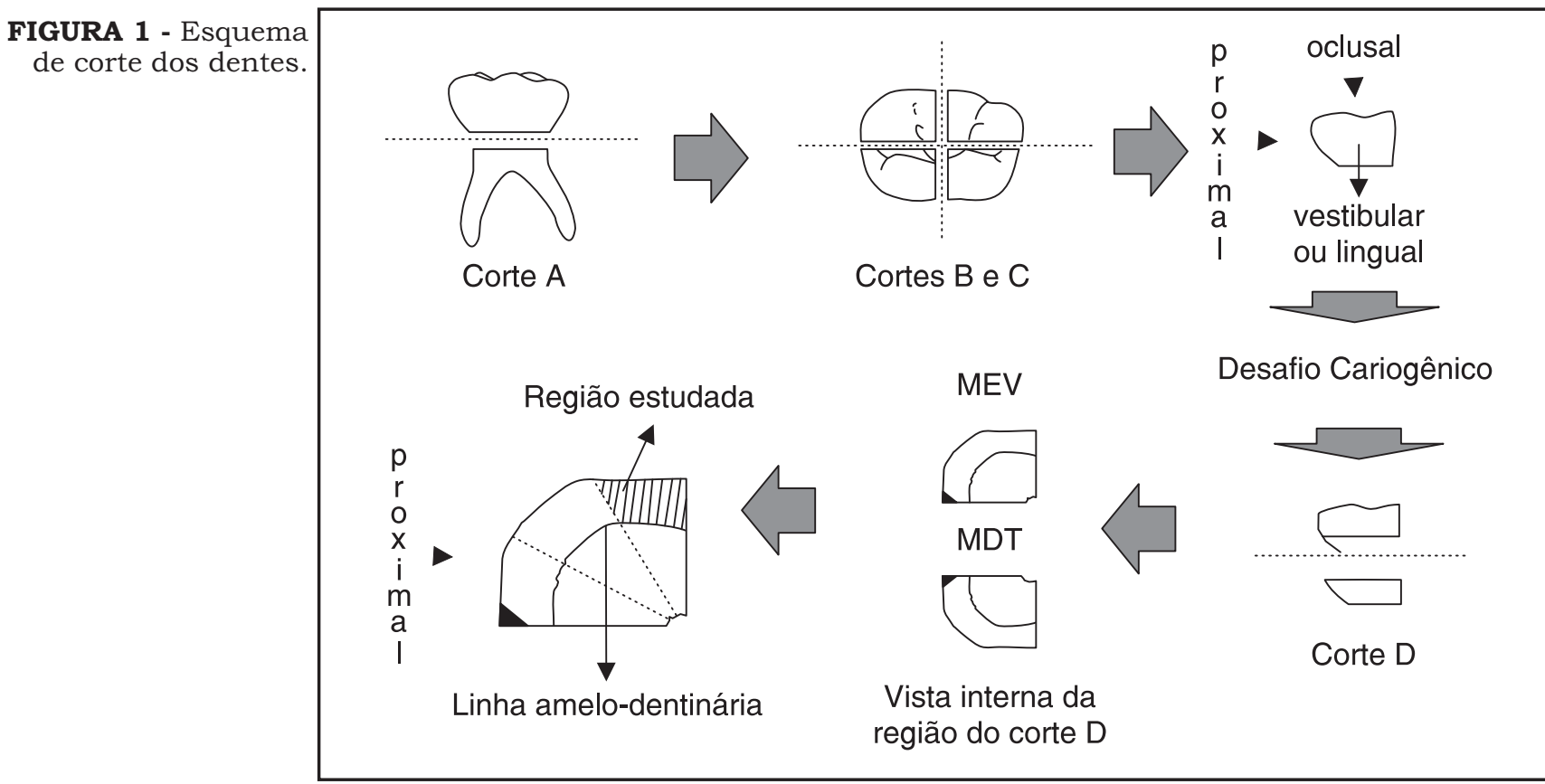


MORAIS, A. P. de; SOUZA, I. P. R. de; CHEVITARESE, O. Estudo in situ do esmalte dental humano após aplicação de tetrafluoreto de titânio. Pesq Odont Bras, v. 14, n. 2, p. 137-143, abr./jun. 2000.

amostra recebeu um escore alfanumérico, composto de uma letra (profundidade da lesão de cárie) e de um número (extensão da lesão de cárie), de acordo com o aspecto da região de esmalte estudado (Figura 2).

Para o estudo de microdureza em corte transversal do esmalte (MDT), as amostras foram embutidas em resina acrílica autopolimerizável de maneira que ficasse exposta a superficie interna do esmalte que foi aplainada e polida. O ensaio de MDT foi realizado por uma Micromet 2003, à qual foi acoplado um penetrador Knoop (25 g/30 s), produzindo cinco marcações com distâncias de $12,5 \mu \mathrm{m}, 25 \mu \mathrm{m}, 50 \mu \mathrm{m}, 75 \mu \mathrm{m}$ e $100 \mu \mathrm{m}$ da super-

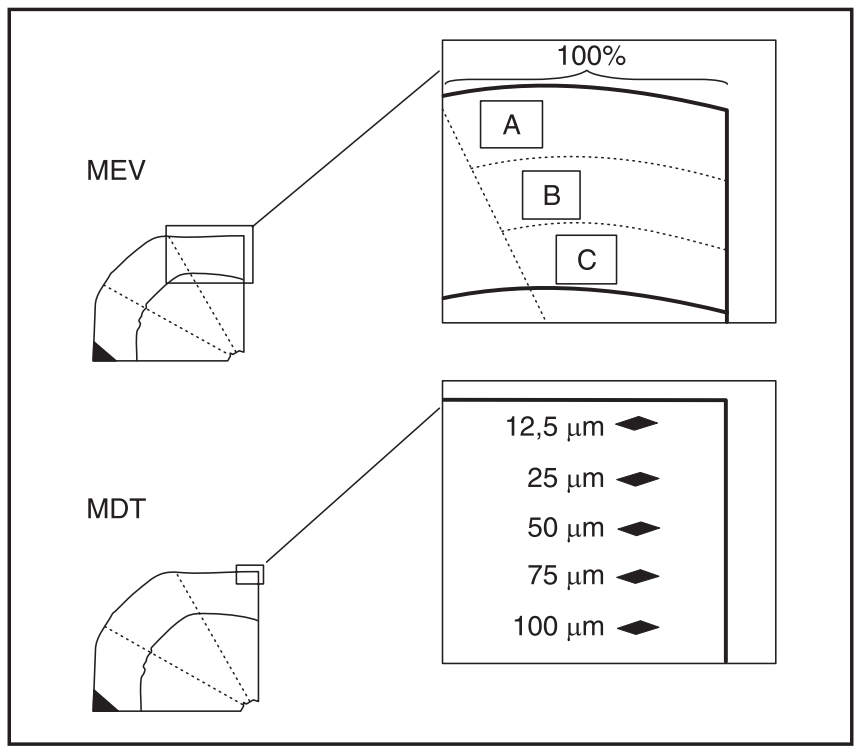

FIGURA 2 - Esquema de análise: MDT e MEV.

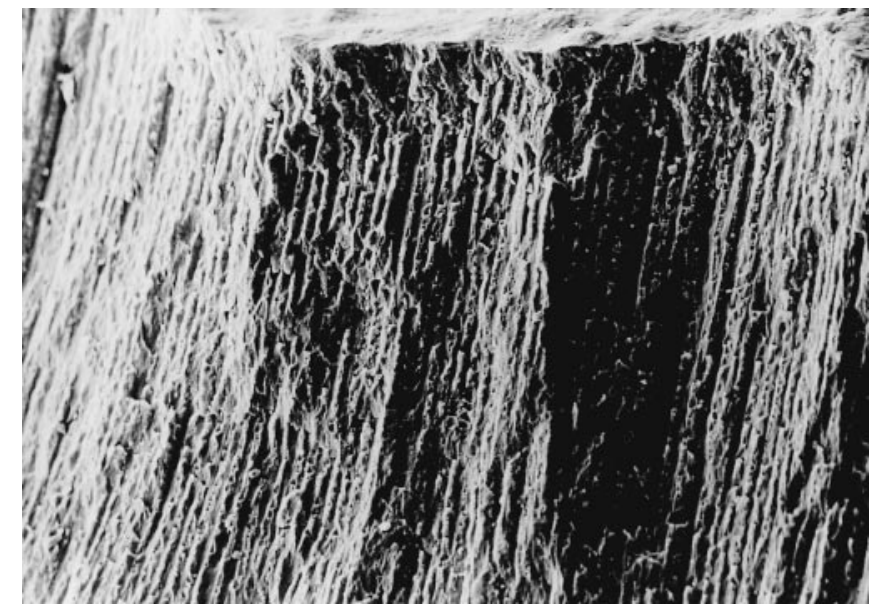

FIGURA 3 - Imagem da MEV referentes ao elemento 38 grupo $\mathrm{TiF}_{4}$, evidenciando esmalte de dente permanente higido $(334 \mathrm{X})$. fície externa do esmalte localizadas a $150 \mu \mathrm{m}$ da bissetriz formada entre a superficie anatômica do esmalte e o corte transversal a ela (Figura 2).

\section{RESULTADOS Microscopia eletrônica de varredura (MEV)}

A análise do esmalte revelou que todos os fragmentos estudados, que possuíam cárie, receberam escore A, ou seja, a lesão cariosa se restringia ao terço superficial do esmalte (Figuras 3, 4 e $5 \mathrm{~A}$ e 5B). Naqueles fragmentos considerados hígidos (10 fragmentos no grupo Controle e 20 fragmentos no grupo $\mathrm{TiF}_{4}$ ), não notou-se a presença de qualquer anormalidade e a preservação das estruturas morfológicas próprias do esmalte ${ }^{8}$. Devido à direção do corte utilizado (corte transversal à superficie) e a posição mediana deste em relação à coroa (Figura 1), havia uma constância na direção apresentada pelos prismas de esmalte. Estes colocavam-se de forma transversal à superficie (Figu$\operatorname{ras} 3,4,5 \mathrm{~A}$ e $5 \mathrm{~B})$.

Nos fragmentos que foram considerados cariados, observou-se regiões de aparência corroída e completa desorganização das estruturas adamantinas onde não era possível visualizar os prismas de esmalte (Figuras 4, 5A e 5B). Somente essas regiões com efeitos intensos de desmineralização foram consideradas cariadas.

Houve uma tendência das lesões, nos fragmentos que possuíam escore 1, se apresentarem como pequenas formações independentes (Figura 4), e nos fragmentos que receberam escore 2 , apresentarem-se contíguas cobrindo toda a superficie externa do esmalte estudado (Figuras 5A e 5B).



FIGURA 4 - Imagem da MEV referente ao fragmento 56 grupo $\mathrm{TiF}_{4}$, evidenciando esmalte de dente decíduo com lesão branda (754 X). 
MORAIS, A. P. de; SOUZA, I. P. R. de; CHEVITARESE, O. Estudo in situ do esmalte dental humano após aplicação de tetrafluoreto de titânio. Pesq Odont Bras, v. 14, n. 2, p. 137-143, abr./jun. 2000.



FIGURA 5A - Imagem da MEV referentes ao fragmento 76 - grupo Controle, evidenciando esmalte de dente permanente com lesão severa (57 X).

Como houve uma igualdade referente à profundidade da lesão, para a análise estatística só foram considerados os dados referentes à extensão da lesão cariosa, designados por: 0 - hígido, 1 - lesão branda e 2 - lesão severa.

Aplicando-se o teste do qui-quadrado ${ }^{10}$ não houve efeito significativo em relação aos voluntários, como também não houve diferença entre dentes decíduos e dentes permanentes. Porém, comparando-se o grupo que recebeu a aplicação da solução de $\mathrm{TiF}_{4}$ com o grupo Controle, ou seja, aquele cujos fragmentos não receberam a aplicação da solução, houve uma diferença significativa onde o valor de $\mathrm{p}$ foi igual a 0,027 (Gráfico 1).

\section{Microdureza em corte transversal do esmalte (MDT)}

Os resultados relativos a MDT foram submetidos à análise de variância multifatorial ${ }^{10} \mathrm{com}$ a mediana dos quatro valores referentes aos quatro fragmentos do mesmo indivíduo em cada etapa. Assim foi verificado efeito significativo apenas em relação aos voluntários e às posições, ou seja, até a profundidade medida, não pôde ser verificada diferença estatisticamente significativa entre os dois grupos.

Considerando os dois grupos $\left(\mathrm{TiF}_{4}\right.$ e Controle), houve diferença estatisticamente significativa entre todas as marcações, com exceção dos pares 50-75 $\mu \mathrm{m}$ e $75-100 \mu \mathrm{m}$, assim pode-se notar, no Gráfico 2 , que as três últimas medianas têm valores bem próximos.



FIGURA 5B - Maior aumento da imagem mostrada na Figura 5A (fragmento 76 - grupo Controle), evidenciando esmalte de dente permanente com lesão severa (486 X).

\section{DISCUSSÃO}

O modelo experimental utilizado permitiu eliminar diferenças intra-indivíduo, pois cada voluntário participou das duas etapas e ao mesmo tempo o desafio cariogênico foi padronizado pelo gotejamento de glicose a $25 \%$, oito vezes ao dia ${ }^{6,15,16}$. Apesar da escolha criteriosa dos voluntários ${ }^{20}$ o resultado da MDT mostrou um efeito significativo para esta variável. A explicação pode estar em diferenças individuais principalmente de placa dental (profundidade, estrutura ou composição microbiana) e/ou em relação às propriedades da saliva que não tiveram uma avaliação específica realizada nesta pesquisa (sistema antimicrobiano e bioquímico complexo). Não houve efeito colateral relatado ou observado entre os voluntários durante ou após o uso dos dispositivos, como também não há relato na literatura odontológica, em todos os ensaios realizados, de nenhum efeito colateral.

Quanto a análise das mudanças no conteúdo mineral do esmalte, os dois métodos, MEV e MDT, não mostraram diferenças significativas entre esmalte de dentes decíduos e de dentes permanentes, talvez pelas características intensas do ataque provocado.

A análise do esmalte através da MDT não mostrou resultado estatisticamente significativo em relação ao uso do $\mathrm{TiF}_{4}$ como agente de prevenção da cárie de esmalte sob severas condições. Porém demonstrou, como já era esperado, que diante de um grande desafio cariogênico, em ambos os grupos, as camadas mais próximas à superfície anatômica do dente foram as mais afetadas, e que, 
MORAIS, A. P. de; SOUZA, I. P. R. de; CHEVITARESE, O. Estudo in situ do esmalte dental humano após aplicação de tetrafluoreto de titânio. Pesq Odont Bras, v. 14, n. 2, p. 137-143, abr./jun. 2000.

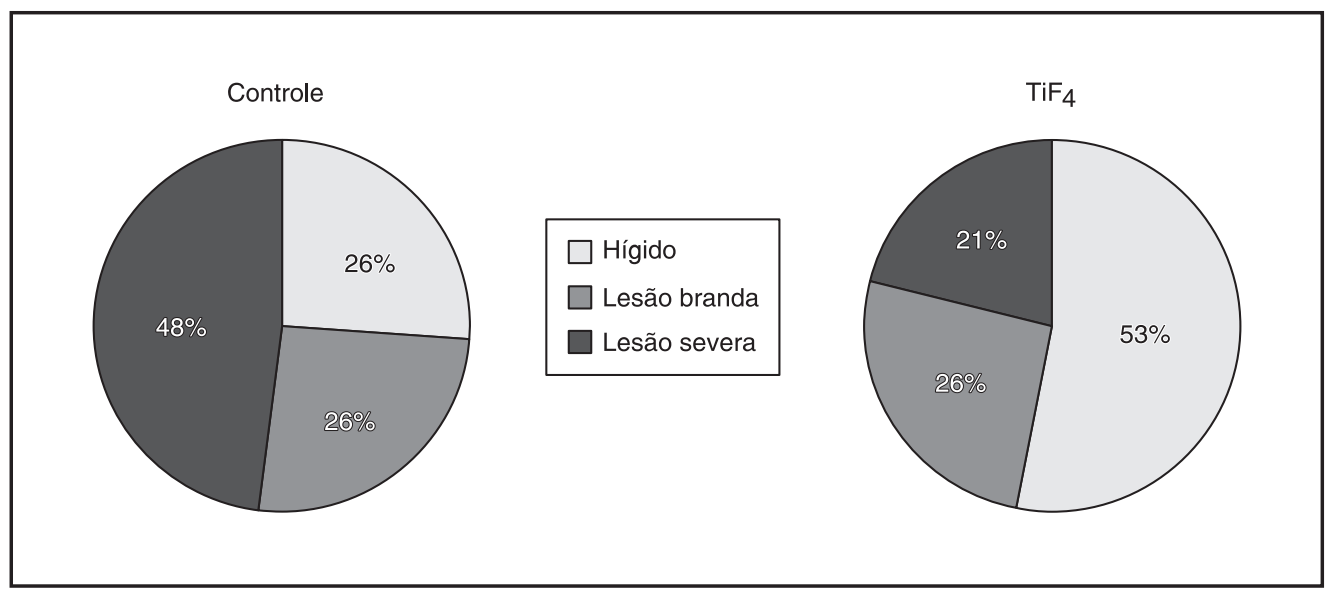

GRÁFICO 1 - Condição do esmalte do grupo Controle e do $\mathrm{TiF}_{4}$ após observação através da MEV. (Obs.: quatro fragmentos foram excluídos.)

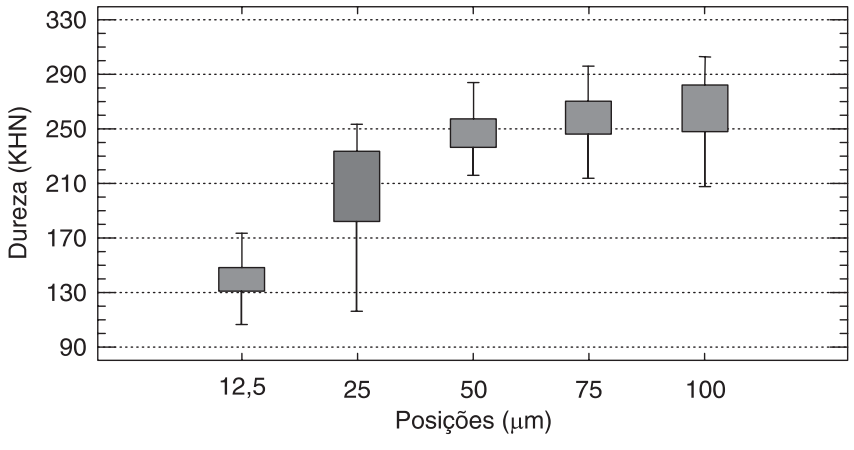

GRÁFICO 2 - Valores da MDT para as 5 marcações (grupo Controle e $\left.\mathrm{TiF}_{4}\right)$.

quanto mais próximo da junção amelodentinária, em especial a partir de $75 \mu \mathrm{m}$, os efeitos do desafio começam a se dissipar (Gráfico 2).

WEFEL ${ }^{19}$ em seu estudo, já argumentava que a aplicação de uma solução de $\mathrm{TiF}_{4}$ não impede a formação da lesão cariosa, mas diminui a profundidade dela cuja camada superficial é rica em fluoreto e titânio, porém pobre em cálcio até a profundidade de $90 \mu \mathrm{m}$, onde os níveis de cálcio sobem drasticamente se comparados aos do esmalte normal. Também BÜYÜKYLMAZ et al. ${ }^{3}$, em trabalho realizado in vivo, diante de condições cariogênicas, encontraram uma diminuição de $37 \%$ na profundidade da lesão formada no grupo $\mathrm{TiF}_{4}$ com $26 \mu \mathrm{m}$ de média de profundidade e 14\% a menos de perda mineral. No grupo Controle, em que nenhum agente anticárie foi aplicado, a média de profundidade das lesões foi de $41 \mu \mathrm{m}$. Na atual pesquisa, a média de dureza Knoop nas camadas mais profundas, aproximou-se, mas não alcançou a exibida por um esmalte hígido, ou seja, acima de 300 "Knoop hardness number" $(\mathrm{KHN})^{14}$. Assim, pode-se supor que, tanto BÜYÜKILMAZ et $a l^{3}$ como WEFEL ${ }^{19}$, obtiveram lesões com profundidades menores que as obtidas neste estudo, tanto para os fragmentos tratados com $\mathrm{TiF}_{4}$ como para seus controles. Essas diferenças nas profundidades das lesões de esmalte formadas, poderiam estar associadas aos diferentes métodos de desmineralização utilizados por eles e conseqüentemente diferente do desafio cariogênico in situ, denotando assim, características de uma acentuada desmineralização neste estudo.

Os resultados obtidos após observações das seções transversais do esmalte através da MEV, mostraram claramente um padrão diferenciado das lesões formadas entre os dois grupos. Enquanto no grupo Controle havia a formação de lesões extensas atingindo linearmente mais de $50 \%$ da superficie anatômica do dente, no grupo $\mathrm{TiF}_{4}$ estas lesões apareciam mais circunscritas. BÜYÜKYLMAZ; SEM; ØGAARD ${ }^{2}$ trabalhando também com seções transversais de esmalte decíduo onde foi aplicado $\mathrm{TiF}_{4}$ a $1 \%$, encontrou zonas de esmalte desmineralizado logo abaixo das superficies observadas pela MEV, sem no entanto, existirem lesões cariosas clinicamente. Estas zonas, que atingiam inclusive ponta de cúspide onde não era possivel visualizar com exatidão os prismas de esmalte, tiveram sua profundidade medida em até $70 \mu \mathrm{m}$.

Uma possível explicação pode estar no fato de o $\mathrm{TiF}_{4}$ ter como produtos de reação presumivelmente o dióxido de titânio e o fluoreto. Este reage com o esmalte, com o ambiente oral ou com a água presente na própria solução formando ácido fluorídrico, caracterizando o baixo $\mathrm{pH}(1,3)$ da solução ${ }^{5}$. Estudos de SHRESTHA; MURDOFF; BIBBY ${ }^{18}$ mostraram que a solução de $\mathrm{TiF}_{4}$ a $1 \%$ dissolve menos esmalte que alguns sucos de frutas e apenas um 
MORAIS, A. P. de; SOUZA, I. P. R. de; CHEVITARESE, O. Estudo in situ do esmalte dental humano após aplicação de tetrafluoreto de titânio. Pesq Odont Bras, v. 14, n. 2, p. 137-143, abr./jun. 2000.

pouco mais que algumas bebidas carbonatadas, porém quando aplicado topicamente em condições clínicas, seu efeito pode ser intensificado levando à produção de zonas de ataque ácido como foi visto no estudo de BÜYÜKYLMAZ; SEM; ØGAARD ${ }^{2}$, já que estas surgiram em regiões do dente relativamente imunes à cárie. Assim, parte das lesões observadas pela $\mathrm{MEV}$, poderiam ser compostas por áreas de desmineralização como aquelas encontradas no trabalho citado ${ }^{2}$, pois ambas são indistinguiveis à observação através da MEV.

\section{CONCLUSÃO}

No presente estudo foi possivel definir que a aplicação tópica de tetrafluoreto de titânio a $1 \%$, sobre esmalte dental decíduo e permanente, possivelmente modifica o padrão de formação da lesão cariosa quando há um grande desafio cariogênico. Porém mais estudos são necessários a fim de que se defina, em que proporções, principalmente em relação a profundidade de lesão, quanto a solução é capaz de exercer este efeito protetor.

MORAIS, A. P. de; SOUZA, I. P. R. de; CHEVITARESE, O. An in situ study of human enamel after titanium tetrafluoride application. Pesq Odont Bras, v. 14, n. 2, p. 137-143, abr./jun. 2000.

\begin{abstract}
The purpose of this in situ, double blind, crossover study was to compare the effectiveness of an application of titanium tetrafluoride $\left(\mathrm{TiF}_{4}\right)$ on deciduous and permanent enamel submitted to highly cariogenic conditions. Five volunteers used palatal appliances with 80 sections of teeth during two phases $\left(\mathrm{TiF}_{4}\right.$ and Control) of 14 days each. They dropped $25 \%$ glucose on the sections, eight times per day. After that, the enamel was analyzed through scanning electron microscopy (SEM) and the Knoop Hardness Number (KHN) was determined at five different depths, up to $100 \mu \mathrm{m}$. The results revealed that differences between deciduous and permanent enamel were not significant. The KHN (Multiple Range Analysis) did not show a significant $\mathrm{TiF}_{4}$ effect, but SEM (Chi Square Test) results revealed differences between $\mathrm{TiF}_{4}$ and the Control group with $\mathrm{p}$ value $=0.027$. There were equal numbers of incipient lesions in both groups, but there were 10 caries-free sections and 18 severe lesions in the Control group, against 20 and 8 in the $\mathrm{TiF}_{4}$ group, respectively. The findings suggested that lesions were less severe with $\mathrm{TiF}_{4}$ and that it changed the pattern of caries, but other studies are necessary to precisely define this effect, in terms of depth.
\end{abstract}

UNITERMS: Fluorides, topical; Titanium; Dental caries.

\section{REFERÊNCIAS BIBLIOGRÁFICAS}

1. BÜYÜKYLMAZ, T.; ØGAARD, B.; DAHM, S. The effect on the tensile bond strength of orthodontic brackets of titanium tetrafluoride $\left(\mathrm{TiF}_{4}\right)$ application after acid etching. Am J Orthod Dentofac Orthop, v. 108, n. 4, p. 256-261, Sept. 1995.

2. BÜYÜKYLMAZ, T.; SEM, B. H.; ØGAARD, B. Retention of titanium terafluoride $\left(\mathrm{TiF}_{4}\right)$, used as fissure sealant on human deciduous molars. Acta Odontol Scand, v. 55, n. 1, p. 73-78, Jan./Fev. 1997.

3. BÜYÜKYILMAZ, T.; TANGUGSORN, V.; ØGAARD, B. et al. The effect of titanium tetrafluoride $\left(\mathrm{TiF}_{4}\right)$ application around orthodontic brackets. Am J Orthod Dentofac Orthop, v. 105, n. 3, p. 293-296, Mar. 1994.

4. CLARKSON, B.; WEFEL, J. Titanium and fluoride concentrations in titanium tetrafluoride and APF treated enamel. J Dent Res, v. 58, n. 2, p. 600-603, Fev. 1979.

5. DUTRA, P. B.; MORAIS, A. P.; VAITSMAN, D. S. Estudo da hidrólise, olação e oxolação do Ti (IV). An Soc Bras Quim Regional Rio de Janeiro. abstr. QA-03, p. 68, 1997.

6. FRANCISCO, S. B.; CURY, J. A. Estudo in situ da relação entre a freqüência de ingestão de sacarose, cárie dental e contagem de estreptococos do grupo mutans na placa dental. An Soc Bras Pesq Odontol, v. 13, abstr. 020, p. 45, 1996.

7. GU, Z.; LI, J.; SÖREMARK, R. Influence of tooth surface conditions on enamel fluoride uptake after topical appli- cation of $\mathrm{TiF}_{4}$ in vitro. Acta Odontol Scand, v. 54, n. 4, p. 279-281, May/Jun. 1996.

8. HUBARD, M. J. Correlated light and scanning electron microscopy of artificial carious lesions. J Dent Res, v. 61, n. 1, p. 14-19, Jan. 1982.

9. JORDAN, T. H.; WEFEL, J. S. The reaction between $\mathrm{TiF}_{4}$ and apatite. J Dent Res, v. 59, spec. iss. A, p. 523, abst. 1017, Nov. 1980.

10. LEVIN, J. Estatística aplicada a ciências humanas. 2. ed. São Paulo : HARBRA, 1987, 392 p.

11. MORAIS, A. P.; BITENCOURT, L.; CHEVITARESE L. A. M. et al. Evaluation of bonding enamel/composite with fresh and olden $\mathrm{TiF}_{4}$ solution. J Dent Res, v. 77, spec. iss. B, abst. 1476, June 1998.

12. MORAIS, A. P.; CHEVITARESE, L. M.; DUTRA, P. D. et al. Colagem esmalte/compósito com ou sem condicionamento e aplicação $\mathrm{TiF}_{4}$. An Soc Bras Pesq Odontol, v. 14, abstr. 052, p. 31, 1997.

13. MURDOFF, S. A.; LITLLE, M. F.; BIBBY, B. G. Enamel dissolution II: action of titanium tetrafluoride. J Dent Res, v. 51, n. 6, Nov./Dec. 1972.

14. PURDELL-LEWIS, D. J.; GROENEVELD, A.; ARENDS, J. Hardness tests on sound enamel and artificially demineralized white spot lesions. Caries Res, v. 10, n. 3, p. 201-215, May/June 1976.

15. REBELlO, M. A. B.; DEL BEL CURY, A. A.; DERBYSHIRE, M. T. C. et al. Composição bioquímica e cariogenicidade da placa dental formada na presença de sacarose ou gli- 
MORAIS, A. P. de; SOUZA, I. P. R. de; CHEVITARESE, O. Estudo in situ do esmalte dental humano após aplicação de tetrafluoreto de titânio. Pesq Odont Bras, v. 14, n. 2, p. 137-143, abr./jun. 2000.

cose + frutose. An Soc Bras Pesq Odontol, v. 15, abstr. A075, p. 30, 1998

16. REBELLO, M. A. B.; DEL BEL CURY, A. A. Incorporação de flúor no esmalte em função da freqüência de exposição à sacarose em região de água fluoretada. An Soc Bras Pesq Odontol, v. 13, abstr. 019, p. 45, 1996.

17. REED, A. J.; BIBBY, G. B. Preliminary report on effect of topical applications of titanium tetrafluoride on dental caries. J Dent Res, v. 55, n. 3 , p. 357-358, May/June, 1976.

18. SHRESTHA, B. M.; MURDOFF, S. M.; BIBBY, B. G. Enamel dissolution I: effect of various agents and titanium tetrafluoride. J Dent Res, v. 51, n. 6, p. 1561-1566, Nov./Dec. 1972.

19. WEFEL, J. S. Artificial lesions formation and fluoride uptake after $\mathrm{TiF}_{4}$ applications. Caries Res, v. 16, n. 1, p. 26-33, Jan. 1982.
20. WEFEL, J. S. Working group report 2: in situ caries models, saliva, microbiology, and statistical considerations. Adv Dent Res, v. 9, n. 3, p. 235-237, Nov. 1995.

21. WEFEL, J. S.; HARLESS, J. D. The effect of topical fluoride agents on artificial lesions formation. J Dent Res, v. 61, n. 10, p. 1169-1171, Oct. 1982.

22. WEFEL, J. S.; HARLESS, J. D. The effect of topical fluoride agents on fluoride uptake and surface morphology. $\mathbf{J}$ Dent Res, v. 60, n. 11, p. 1842-1848, Nov. 1981.

23. WEFEL, J. S.; VALERIO, J.; WEI, S. H. Y. TiF 4 as a topical fluoride agent. J Dent Res, v. 56, spec. iss. B, abst. 321, Nov. 1977.

24. WEI, S. H. Y.; SOBOROFF, D. M.; WEFEL, J. S. Effects of titanium tetrafluoride on human enamel. J Dent Res, v. 55, n. 3, p. 426-431, May/June 1976.

Recebido para publicação em 06/01/00 Enviado para reformulação em 20/03/00 Aceito para publicação em 03/05/00 